\title{
Water Mediated Green Synthesis of Coumarin-3-carboxylic Acids under Microwave Irradiation Condition
}

\author{
DINESH SHARMA*, SURENDER KUMAR and J K MAKRANDI \\ *Department of Chemistry, BRCM College of Engineering \& Technology, \\ Bahal, 127028, India \\ Department of Chemistry, Technological Institute of Textile \& Sciences, \\ Bhiwani, 127021, India \\ Department of Chemistry, M. D University, Rohtak, 124001, India. \\ dksharma_84@rediffmail.com
}

Received 17 August 2012 / Accepted 19 September 2012

\begin{abstract}
A simple, efficient and green procedure for the synthesis of coumarin-3-carboxylic acids has been developed which involves the reaction of 2-hydroxybenzaldehydes with Meldrum's acid (2,2-dimethyl1,3-dioxan-4,6-dione) in aqueous moist conditions under microwave irradiation. The protocol is much more efficient as the reactions were carried out at milder conditions and yields were also quite high.
\end{abstract}

Keywords: Coumarin-3-carboxylic acid, 2-Hydroxybenzaldehyde, Meldrum's acid, Microwave condition, Green synthesis

\section{Introduction}

Coumarin-3-carboxylic acids (3-carboxycoumarins) constitute an important class of compounds because of their vast applications. These are required intermediates for the synthesis of number of natural products with diverse biological activities ${ }^{1}$. Further these compounds have been used for the synthesis of modified cephalosporins ${ }^{2}$, pencillins ${ }^{3}$, isoureas ${ }^{4}$, oxygen-bridged tetrahydropyridones ${ }^{5}$, compounds with specific inhibition activity of $\alpha$-chymotrypsin and human Leukocyte elastase ${ }^{6,7}$.

In recent studies, coumarin-3-carboxylic acid derivatives have been found to be potent and selective inhibitors to monoamine oxidase and showed marked potency in inhibiting cancer cell invasion in vitro and tumor growth in vivo ${ }^{8-10}$. Their metal complexes have also been found to exhibit good biological properties ${ }^{11,12}$.

Coumarin-3-carboxylic acids have also been used as fluorescent probes and triplet sensitizers ${ }^{13,14}$ and also display wide application in perfume and cosmetic industry ${ }^{15}$. Due to their important role in varied fields, lot of emphasis has been laid in their synthesis ${ }^{16-18}$. Initially, these compounds were obtained by condensation of substituted salicylaldehyde with malonic acid, ethylcyanoacetate, malononitrile $e^{19-23}$ in presence of piperidine ${ }^{24}$, piperidine acetate $^{25}$, ammonium acetate ${ }^{26}$, sulphuric acid adsorbed in silica ${ }^{27}, L$-proline ${ }^{28}$ and ionic liquids ${ }^{29}$. 
Use of Meldrum's acid was found to be much superior in terms of yields. In recent reports, these have been obtained by condensation of salicylaldehyde with Meldrum's acid under phase transfer catalysed condition using triethyl benzyl ammonium chloride $(\text { TEBAC })^{30}$ and potassium phosphate in ethanol ${ }^{31}$.

In continuation of our work on development of eco-friendly synthesis of organic compounds using aqueous medium ${ }^{32}$, we wish to report a simple and efficient protocol for the synthesis of coumarin-3-carboxylic acids.

\section{Experimental}

All the chemicals were purchased from Aldrich and Fluka. Melting points were determined in open capillaries. The reactions were carried out in a domestic Samsung microwave oven with output energy $900 \mathrm{~W}$, frequency $2450 \mathrm{MHz}$ with temperature control arrangement model no. CE1031LFB.

\section{General procedure}

A mixture of 2-hydroxybenzaldehydes (1, $4.1 \mathrm{mmol})$ and Meldrum's acid (2, 4.16 mmol) moist with 5 drops of water was subjected to microwave irradiations using $50 \%$ power of microwave oven for 2 minutes $\left(12 \times 10\right.$ seconds) at $100{ }^{\circ} \mathrm{C}$. The completion of the reaction was checked by thin layer chromatography. The reaction mixture was cooled to room temperature and diluted with ice-cold water. The solid that separated out was filtered at vacuum, washed with water and recrystallised from ethanol to give coumarin-3-carboxylic acids.

\section{Spectral characterization of compounds}

Coumarin-3-carboxylic acid (3a)

mp 190-191 ${ }^{\circ} \mathrm{C}$; IR (KBr) 3415 (OH), 1745 (C=O), 1685 (C=O) $\mathrm{cm}^{-1}$; ${ }^{1} \mathrm{H}$ NMR (400 MHz, $\left.\mathrm{CDCl}_{3}\right) \delta$ 7.42-7.82 (m, 3H, H-6, H-7, H-8), 7.92-7.94 (dd, $J=8.0 \mathrm{~Hz} \& J=2.0 \mathrm{~Hz}, 1 \mathrm{H}, \mathrm{H}-5$ ), 8.74 (s, 1H, H-4), 13.25 (s, 1H, COOH).

\section{6-Bromocoumarin-3-carboxylic acid (3b)}

mp 193-195 ${ }^{\circ} \mathrm{C}$; IR (KBr) $3310(\mathrm{OH}), 1742(\mathrm{C}=\mathrm{O}), 1712$ (C=O) $\mathrm{cm}^{-1}$; ${ }^{1} \mathrm{H}$ NMR (400 MHz, $\left.\mathrm{CDCl}_{3}\right) \delta 7.65$ (d, $\left.J=8.0 \mathrm{~Hz}, 1 \mathrm{H}, \mathrm{H}-8\right), 7.80-7.82$ (dd, $J=8.0 \mathrm{~Hz} \& J=2.0 \mathrm{~Hz}, 1 \mathrm{H}, \mathrm{H}-7$ ), 8.10 (d, $J=2.0 \mathrm{~Hz}, 1 \mathrm{H}, \mathrm{H}-5$ ), 8.45 (s, 1H, H-4), 12.95 (s, 1H, COOH).

\section{6-Chlorocoumarin-3-carboxylic acid (3c)}

mp 120-122 ${ }^{\circ} \mathrm{C}$; IR (KBr) $3195(\mathrm{OH}), 1748(\mathrm{C}=\mathrm{O}), 1678(\mathrm{C}=\mathrm{O}) \mathrm{cm}^{-1}$; ${ }^{1} \mathrm{H}$ NMR (400 MHz, $\left.\mathrm{CDCl}_{3}\right) \delta 7.48(\mathrm{~d}, J=8.0 \mathrm{~Hz}, 1 \mathrm{H}, \mathrm{H}-8), 7.73-7.75$ (dd, $\left.1 \mathrm{H}, J=8.0 \& J=2.0 \mathrm{~Hz}, \mathrm{H}-7\right), 7.95$ (d, $J=2.0 \mathrm{~Hz}, 1 \mathrm{H}, \mathrm{H}-5), 8.82$ (s, 1H, H-4), 13.65 (s, 1H, COOH).

6-Methylcoumarin-3-carboxylic acid (3d)

mp $165{ }^{\circ} \mathrm{C}$; IR (KBr) $3150(\mathrm{OH}), 1725(\mathrm{C}=\mathrm{O}), 1686(\mathrm{C}=\mathrm{O}) \mathrm{cm}^{-1}$; ${ }^{1} \mathrm{H}$ NMR (400 MHz, $\left.\mathrm{CDCl}_{3}\right) \delta 2.45$ (s, 3H, $\mathrm{CH}_{3}$ ), 7.18 (d, $J=8.0 \mathrm{~Hz}, 1 \mathrm{H}, \mathrm{H}-8$ ), 7.20-7.23 (dd, $J=8.0 \mathrm{~Hz} \& J=2.0$ Hz, 1H, H-7), 7.38 (d, J=2.0 Hz, 1H, H-5), 8.60 (s, 1H, H-4), 12.92 (s, 1H, COOH).

\section{6-Methoxycoumarin-3-carboxylic acid (3e)}

mp 208-209 ${ }^{\circ} \mathrm{C}$; IR (KBr) $3152(\mathrm{OH}), 1726(\mathrm{C}=\mathrm{O}), 1688(\mathrm{C}=\mathrm{O}) \mathrm{cm}^{-1}$; ${ }^{1} \mathrm{H}$ NMR (400 MHz, $\left.\mathrm{CDCl}_{3}\right) \delta 3.23$ (s, 3H, $\mathrm{OCH}_{3}$ ), 7.15 (d, $J=8.0,1 \mathrm{H}, \mathrm{H}-8$ ), 7.35-7.38 (dd, $J=8.0 \mathrm{~Hz} \& J=2.0 \mathrm{~Hz}$, 1H, H-7), 7.42 (d, J=2.0 Hz, 1H, H-5), 8.62 (s, 1H, H-4), 12.95 (s, 1H, COOH). 
7-Methoxycoumarin-3-carboxylic acid (3f) mp 175-177 ${ }^{\circ} \mathrm{C}$; IR (KBr) $3320(\mathrm{OH}), 1738(\mathrm{C}=\mathrm{O}), 1682(\mathrm{C}=\mathrm{O}) \mathrm{cm}^{-1}$; ${ }^{1} \mathrm{H}$ NMR (400 MHz, $\left.\mathrm{CDCl}_{3}\right) \delta 3.90\left(\mathrm{~s}, 3 \mathrm{H}, \mathrm{OCH}_{3}\right), 7.05-7.15(\mathrm{~m}, 2 \mathrm{H}, \mathrm{H}-6, \mathrm{H}-8), 7.86$ (d, J=8.0 Hz, 1H, H-5), 8.75 (s, 1H, H-4), 12.85 (s, 1H, COOH).

\section{5,7-Dimethoxycoumarin-3-carboxylic acid (3g)}

mp 233-235 ${ }^{\circ} \mathrm{C}$; IR (KBr) $3345 \mathrm{~cm}^{-1}(\mathrm{OH}), 1748(\mathrm{C}=\mathrm{O}), 1682(\mathrm{C}=\mathrm{O}) \mathrm{cm}^{-1}$; ${ }^{1} \mathrm{H}$ NMR (400 $\left.\mathrm{MHz} \mathrm{CDCl}_{3}\right) \delta 3.88$ (s, 3H, $\mathrm{OCH}_{3}$ ), 3.92 (s, 3H, $\mathrm{OCH}_{3}$ ), 6.52 (d, J=2.0 Hz, 1H, H-8), 6.64 (d, J=2.0 Hz, 1H, H-6), 8.55 (s, 1H, H-4), 12.96 (s, 1H, COOH).

\section{Results and Discussion}

In a typical experimental procedure, a mixture of 2-hydroxybenzaldehyde and Meldrum's acid was taken in a loosely stoppered round bottom flask moist with 5 drops of water and was subjected to microwave irradiations using $50 \%$ power of microwave oven for 2 minutes (12x10 seconds) at $100{ }^{\circ} \mathrm{C}$ (Scheme 1 ). The progress of the reaction was monitored by thin layer chromatography. The reaction was worked up by diluting with ice cold water to give coumarin-3-carboxylic acid whose structure was confirmed by its spectral data. The validity of the procedure was checked by preparing differently substituted coumarin-3-carboxylic acids. The identity of the compounds (Table 1) was confirmed from their IR, ${ }^{1} \mathrm{H}$ NMR spectra and melting point comparison with literature value.<smiles>[R]c1cc(O)c(C=O)c([12CH]I)c1[R]</smiles><smiles>CC1(C)OC(=O)CC(=O)O1</smiles>

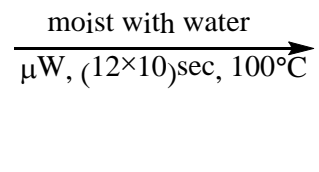<smiles></smiles>

Scheme 1. Synthesis of conmarin-3-carboxylic acids

Table 1. Physical properties of coumarin-3-carboxylic acids

\begin{tabular}{clllccc}
\hline Compound & $\mathrm{R}_{1}$ & $\mathrm{R}_{2}$ & $\mathrm{R}_{3}$ & Time, min & Yield, \% & M.Pt, ${ }^{\circ} \mathrm{C}$ \\
\hline 3a & $\mathrm{H}$ & $\mathrm{H}$ & $\mathrm{H}$ & 2 & 85 & $190-191(191-192)^{25}$ \\
3b & $\mathrm{H}$ & $\mathrm{Br}$ & $\mathrm{H}$ & 2 & 90 & $193-195(199)^{33}$ \\
3c & $\mathrm{H}$ & $\mathrm{Cl}$ & $\mathrm{H}$ & 2 & 80 & $120-122(120-121)^{25}$ \\
3d & $\mathrm{H}$ & $\mathrm{CH}_{3}$ & $\mathrm{H}$ & 2 & 88 & $165(166-167)^{34}$ \\
3e & $\mathrm{H}$ & $\mathrm{OCH}_{3}$ & $\mathrm{H}$ & 2 & 86 & $208-209(206-207)^{34}$ \\
3f & $\mathrm{OCH}_{3}$ & $\mathrm{H}$ & $\mathrm{H}$ & 2 & 85 & $175-177(177)^{33}$ \\
3g & $\mathrm{OCH}_{3}$ & $\mathrm{H}$ & $\mathrm{OCH}_{3}$ & 2 & 80 & $233-235(234-237)^{33}$ \\
\hline
\end{tabular}

\section{Conclusion}

The present synthesis of coumarin-3-carboxylic acids in aqueous medium under microwave irradiation condition is fairly clean, rapid and efficient. This protocol is an eco-friendly one as it avoids the use of hazardous organic solvents at any stage of the reaction.

\section{References}

1. Murray R D H, Mendez J and Brown S A, The Natural Coumarins, John Wiley \& Sons: New York, 1977. 
2. Bonsignore L, Cittiglia F, Elkhaili H, Jehl F, Lavagna S M, Loy G, Manna F, Monteil H, Pompei D and Secci D, Farmoco, 1998, 53(6), 425-430.

3. Bonsignore L, De Logu A, Lavagna S M, Loy G and Secci D, Eur J Med Chem., 1994, 29, 479-485.

4. Bonsignore L, Cottiglia F, Lavagna S M, Loy G and Secci D, Heterocycles, 1999, 50, 469-478.

5. Jonsson D, Erlandsson M and Unden A, Tetrahedron Lett., 2001, 42, 6953-6956.

6. Pochet L, Doucet C, Thierry N, Schynts M, Boggeto N and Pirotte B, J Med Chem., 1996, 39, 2579-2585.

7. Doucet C, Pochet L, Thierry N, Pirotte B, Delarge J and Rebound R M, J Med Chem., 1999, 42(20), 4161-4171.

8. Chimenti F, Secci D, Bolasco A, Chimenti P, Granese A, Befani O, Turini P, Alcaro S and Ortuso F, Bioorg Med Chem Lett., 2004, 14, 3697-3703.

9. Kempen I, Hemmer M, Counerotte S, Pochet L, de Tullio P, Foidart J M, Blacher S, Noe"l A, Frankenne F and Pirotte B, Eur J Med Chem., 2008, 43(12), 2735-2750.

10. Kempen I, Papapostolou D, Thierry N, Pochet L, Counerotte S, Masereel B, Foidart J M, Reboud-Ravaux M, Noel A and Pirotte B, Br J Cancer, 2003, 88(7), 1111-1118.

11. Karaliota A, Kretsi O and Tzougraki C, J Inorg Biochem., 2001, 84, 33-37.

12. Creaven B S, Egan D A, Kavanagh K, McCann M, Noble A, Thati B and Walsh M, Inorg Chim Acta, 2006, 359, 3976-3984.

13. Peroni E, Caminati G, Baglioni P, Nuti F, Chelli M and Papini A M, Bioorg Med Chem Lett., 2002, 12, 1731-1734.

14. Specht D P, Martic P A and Farid S, Tetrahedron, 1982, 38(9), 1203-1211.

15. Meuly W C, K-Othmer Encylopedia of Chemical Technology, $3{ }^{\text {rd }}$ Ed., John Wiley \& Sons: New York, 1979, 7, 196-206.

16. Wiener C, Schroeder C H and Link K P, J Am Chem Soc., 1957, 79, 5301-5303.

17. Watson B T and Christiansen G E, Tetrahedron Lett., 1998, 39, 6087-6090.

18. Bigi F, Chesini L, Maggi R and Sartori G, J Org Chem., 1999, 64, 1033-1035.

19. Maggi R, Bigi F, Carloni S, Mazzacani A and Sartori G, Green Chem., 2001, 3, 173-174.

20. Shirokova E A, Segal G M and Torgov I V, Bioorganicheskaya Khimiya, 1988, 14, 236-242.

21. Bandgar B P, Uppalla L S and Kurule D S, Green Chem., 1999, 1, 243-245.

22. Bandgar B P, Uppalla L S and Sadavarte V S, J Chem Res S, 2002, 40-41.

23. Waston B T and Christiansen G E, Tetrahedron Lett., 1998, 39, 6087-6090.

24. Creaven B S, Egan D A, Karcz D, Kavanagh K D, McCann M, Mahon M, Noble A, Thati B and Walsh M, J Inorg Biochem., 2007, 101(8), 1108-1119.

25. Song A, Wang X and Lam K S, Tetrahedron Lett., 2003, 44, 1755-1758.

26. Scott J L and Raston C L, Green Chem., 2000, 2, 245-247.

27. Hekmatshoar R, Rezaei A and Bheshtiha S Y S, Phosphorus, Sulfur Silicon Relat. Elem., 2009, 184, 2491-2496.

28. Karade N N, Gampawar S V, Shinde S V and Jadhav W N, Chin J Chem., 2007, 25, 1686-1689.

29. Darvatkar N B, Deorukhkar A R, Bhilare S V, Raut D G and Salunkhe M M, Synth Commun., 2008, 38(20), 3508-3513.

30. Yang L, Xiyue Tao, Guihai Lin and Gao W, Synth Commun., 2012, 42, 2067-2074.

31. Undale K A, Gaikwad D S, Shaikh T S, Desai U V and Pore D M, Indian J Chem., 2012, 51B, 1039-1042.

32. Jakhar K and Makrandi J K, Green Chem Lett Rev., 2008, 1, 219-221.

33. Fringuelli F, Piermatti O and Pizzo F, Synthesis, 2003, 15, 2331-2334.

34. Bigi F, Chesini L, Maggi R and Sartori G, J Org Chem., 1999, 64, 1033-1035. 